\title{
Preparation of graphene oxide reinforced hydroxyapatite Poly (vinyl alcohol) nanocomposite materials
}

\author{
Qi Cheng ${ }^{\# 1,2}$, Qian Lan ${ }^{\# 1,2}$, Chang Liu ${ }^{1,2}$, Jinxing Zhao ${ }^{1,2}$,Jiyuan Liang ${ }^{1,2}$, Shun Tang ${ }^{1,2}$, and Yuan-Cheng Cao ${ }^{* 1,2}$ \\ ${ }^{1}$ Key Laboratory of Optoelectronic Chemical Materials and Devices of Ministry of Education, Jianghan University, Wuhan 430056, China \\ ${ }^{2}$ Flexible Display Materials and Technology Co-Innovation Centre of Hubei Province, Jianghan University, Wuhan 430056, China \\ \#These authors contributed equally to this work.
}

\begin{abstract}
In this paper, graphite oxide and hydroxyapatite (HA) were successfully synthesized. GO was used to improve the barrier property and mechanical strengthen for the nanocomposite materials. XRD showed that the GO diffraction peak for the corresponding (001) reflection is about $2 \theta=12.1^{\circ}$ and AFM observations showed that the GO sheets heighted around $400 \mathrm{~nm}$. SEM observation of HA nanoparticles indicates that the morphology of the HA was rod like particle with 30-50 nm in length and 10-20 nm wide and HA-GO SEM showed that the GO flasks covered with the HA particles to form the uniform mixtures. FTIR spectrum of HAGO-PVA, these vibrations combined the results from HA, GO and PVA. DSC thermograms indicates that the glass transition temperature ( $\mathrm{T}_{\mathrm{p}}$ ) for the HA-GOPVA nanocomposite was $78^{\circ} \mathrm{C}$. Thermogravimetric analysis (TGA) showed three steps of weight loss: $13.3 \%$ absorbed water release from $70{ }^{\circ} \mathrm{C}$ to $215^{\circ} \mathrm{C} ; 52.4 \%$ of PVA polymer chains degradation from $215^{\circ} \mathrm{C}$ to $410{ }^{\circ} \mathrm{C}$; and $14.2 \%$ further dehydrated of $\mathrm{HA} 410{ }^{\circ} \mathrm{C}$ to $526{ }^{\circ} \mathrm{C}$. The stress-strain curve for the HA-GO-PVA nanocomposite showed that the tensile strength was $42.6 \mathrm{MPa}$ and Young's modulus was $207.8 \mathrm{MPa}$, and the fractured elongation was $130 \%$. These results showed that this nanocomposite material is a promising coating material for the biomedical applications.
\end{abstract}

\section{Introduction}

Nanomaterials which consist of at less one dimension in nano scale, have simulated considerable attention in the research field of advanced materials. At the same time, hydroxyapatite (HA) with formation of $\mathrm{Ca}_{10}(\mathrm{PO} 4)_{6}(\mathrm{OH})_{2}$, is a biomaterial that commonly used in surgical applications including bone substitution and bone regeneration because of its excellent biocompatibility, high bioactivity, and good osseoconductivity. Due to the developments of nanotechnologies, considerable research efforts have been attracted to develop HA nanomaterials for suitable bone and teeth substitutes because of their similarities with inorganic mineral components in human bodies [1-6]. A number of applications have been reported for the nanoscale HA in various fields in column chromatography in life science, ceramic reinforcement in medical science, bone defect filler, ion exchange of heavy metal, and amino acids purifications by virtue of their own physical properties depending on morphologies [5-10]. HA based polymer nanocomposites are one of these promising nanomaterials which take the advantages of nanotechnologies in the materials researches. Changing of the matrix and nano-dimensional phase(s) results in novel properties due to the dissimilarities in structure and chemistry which are remarkable different from that of the original component materials [11-14], not only in the reinforcement due to the high area to volume ratio, but also in the physical and engineering properties including biocompatibility, high bioactivity, and barrier [13-18].

HA has also been used as coatings in load-bearing metallic implants because of its osseoconductivity and chemical similarity to bone which needs to be addressed considering the long-term stability and performance of HA coatings. Clinical successes of HA coated metallic implants are well documented [13, 17, 19]. To date, extensive research has been done to investigate the effects of organic molecules on HA surgery applications [17-20]. The results showed that a variety of polymers, metals can be applied to synthesize HA based composite materials for the biomedical applications. For example, hydroxyapatite- $\mathrm{TiO}_{2}$-based nanocomposites synthesized in supercritical CO2 for bone tissue engineering [11], bioartificial bone tissue poly-L-lactic acid/polycaprolactone [3], antibacterial properties of a hydroxyapatite adhesive block copolymer [5], poly (1-lactic acid)grafted hydroxyapatite/poly (lactic-co-glycolic acid) scaffolds [10], poly ( $\varepsilon$-caprolactone)/ hydroxyapatite thin films [17], zirconium nitride and hydroxyapatite nanocomposite coating [19]. Polyvinyl alcohol (PVA) is completely composed of ethylene oxygen groups $(-\mathrm{CH} 2-\mathrm{CH}(\mathrm{OH})-)$ and has been widely used in biomedical research and applications. PVA of different molecular weights have attracted intensive interest in drug delivery systems and bone tissue engineering.

On another hand, graphite oxide (GO), formerly called graphitic oxide or graphitic acid, is a compound of carbon, oxygen, and hydrogen in variable ratios. GO is widely used as barrier fillers in the polymer materials and used as mechanical strengthen fillers as well [18]. Hydrophilic graphene oxide disperses readily in most of the polar polymer matrix and it can effectively improve the resultant material mechanical strength. GO and HA provide insights into the

Correspondence to: Yuan-Cheng Cao, Key Laboratory of Optoelectronic Chemical Materials and Devices of Ministry of Education, Jianghan University, Wuhan 430056, China, Tel.: + (0)86-27-5125 3048, E-mail: yuancheng.cao@ jhun.edu.cn

Key words: Hydroxylapatite, graphene oxide, bone regeneration, biocompatibility, nanomaterials, barrier property

Received: January 28, 2017; Accepted: February 22, 2017; Published: February 24, 2017 
development of GO/HA based polymers nanocomposite coatings, and has facilitated materials design from biomedical point view.

As graphite oxide/HA nanocomposites have many promising properties such as biocompatibility in medical science, and people are interested to develop biomaterials with coating capacity and having good mechanical strength. Here in this work, we are trying to use the graphite oxide/HA based Poly (vinyl alcohol) (PVA) nanocomposite coating to study the feasibility of biocompatibable coatings and hope this could develop a high efficient nanocomposite for the medical applications.

\section{Results and discussions}

Graphene Oxide (GO) contains a range of reactive groups such as carboxyl and oxygen, and these groups make it a good candidate for various applications including polymer composites materials. Though the detailed structure is still not completely clear due to the amorphous character, a number of applications involving barrier property, mechanical strengthen have been developed over the development of materials chemistry, and many of these applications can be applied to biomedical nanocomposite materials.

From the results, we can see that the typical graphene diffraction peak is about $2 \theta=27^{\circ}$ corresponding to the (001) plane reflection of GO can be observed from the spectra of graphene [18]; while in the spectra of $\mathrm{GO}$, the diffraction peak for the corresponding (001) reflection is about $2 \theta=12.1^{\circ}$ (Figure1), which means that the layer distance is much higher than of the graphite. AFM observations in Figure 1 (B) showed that GO particles were in the size of $400 \mathrm{~nm}$ in height. The resultant bulk product is yellow solid that has the typical diffraction reflection peak (001), indicating that GO retains the layer structure of graphite.

Hydroxyapatite (HA) is chemically compatible with the inorganic phase of natural bone and has been widely used in the field of biomaterials. The SEM observation of as-synthesized HA nanoparticles are shown in Figure 2 (A), which indicates that the morphology of the HA was rod like particle with $30-50 \mathrm{~nm}$ in length and $10-20 \mathrm{~nm}$ wide. The size is similar to the size of HA in natural bones [22-24]. SEM observation on the HA-GO samples showed that the GO flasks covered with the HA particles to form the uniform mixtures Figure 2 (B). In this mixture, the GO is a good dispersant which stops the aggregation of HA nanoparticles.

The PVA based HA-GO composite materials were further tested by the FTIR, DSC, TGA and mechanical stress. FTIR studies in Fig.3 confirmed the successful oxidation of graphite to graphite oxide which showed at $1620 \mathrm{~cm}^{-1}$ (stretching vibrations from $\mathrm{C}=\mathrm{O}$ or $\mathrm{COOH}$ ) [18]. The FTIR spectrum of HA in Figure 3 shows the presence of at 636 $\mathrm{cm}^{-1}$ (O-H stretching vibrations), and $1020 \mathrm{~cm}^{-1}\left(\mathrm{CaCO}_{3}\right.$ stretching vibrations). And in the spectrum of HA-GO-PVA, these vibrations combined the results from HA, GO and PVA.

Differential scanning calorimetry (DSC) and thermogravimetric analysis (TGA) were applied to characterize the HA-GO-PVA composites, and data are shown in Figure 4 (A) and (B), respectively. The DSC thermograms in $\mathrm{N}_{2}$ flow indicates that the glass transition temperature $\left(\mathrm{T}_{\mathrm{g}}\right)$ for the HA-GO-PVA nanocomposite was $78{ }^{\circ} \mathrm{C}$ (Figure 4 (A), which indicated that the HA and GO formed steady composite in the PVA polymer matrix.

Thermogravimetric analysis (TGA) was applied to characterize the HA-GO-PVA nanocomposite and data are shown in Figure 3 (B). The TGA curve showed three steps of weight loss: absorbed water release from $70^{\circ} \mathrm{C}$ to $215^{\circ} \mathrm{C}$ which counted $13.3 \%$ of the weight; PVA polymer chains degradation from $215^{\circ} \mathrm{C}$ to $410^{\circ} \mathrm{C}$ which counted $52.4 \%$; and further dehydrated of HA $410{ }^{\circ} \mathrm{C}$ to $526^{\circ} \mathrm{C}$ which counted $14.2 \%$.

Tensile tests were carried out the nanocomposite materials to determine the mechanical properties and the results are shown in Figure 5. From the results, we can see that the HA-PVA nanocomposites only had the tensile strength was 8.1 MPa and Young's modulus was $77.8 \mathrm{MPa}$, and the fractured elongation was $91 \%$. While the stressstrain curve for the HA-GO-PVA nanocomposite showed that the tensile strength was $42.6 \mathrm{MPa}$ and Young's modulus was $207.8 \mathrm{MPa}$, and the fractured elongation was $130 \%$. These results indicate that the HA-GO in the PVA matrix was compatible to form the uniform nanocomposite and the GO can improve the mechanical strength. This may be caused by the -OH groups in the HA and GO with the PVA polymer matrix which are hydrophilic so that the mechanical strength can be maintained after the addition of the HA and GO.

The HA-GO-PVA was applied to study the application of the biomedical coatings for the bone implants and teeth replacements. As showed in the Figure 6 (A), the nanocomposite was cast onto the glass plate showed good coating ability and SEM surface observations showed the surface with HA-GO which is suitable for the tissue growth Figure 6 (B) and cross-section SEM showed the coating formed uniform coatings Figure $6(\mathrm{C})$. These results showed that this nanocomposite material is a promising coating material for the biomedical applications.

\section{Experimental details}

\section{Chemicals and reagents}

Expandable graphene (EG) (100nm thick) was purchased from Shandong Qingdao Company limited (China), grade ES/350/ FS. Poly (vinyl alcohol) (PVA) was purchased from supermarket. Sulphuric acid, ethanol, and potassium dihydrogen phosphate, ammonium dihydrogen phosphate, Calcium nitrate, hydrogenperoxide, and dimethyl sulfate (DMS, catalogue no. D186309), Dimethyl sulfoxid (DMSO) were purchased from Sigma Aldrich.

\section{Graphene oxide preparation}

Graphene oxide preparation was referred to the references ${ }^{18}$ using the sulphuric acid and Potassium permanganate. Briefly, $60 \mathrm{~mL}$ concentrated sulphuric acid was cooled to $5^{\circ} \mathrm{C}$ and then 10 grams of Graphite powder was added into the cold sulphuric acid with constant stirring. Once the Graphite was completely mixed, 20 grams of Potassium permanganate was then added slowly to the solution. Then the mixture was stirred for another 5-10 minutes in the ice bath. The mixture was then heated up to $35-40^{\circ} \mathrm{C}$. This resulted in the formation of a thick black paste. $150 \mathrm{~mL}$ of distilled water was then added drop wise to the overall mixture. This mixture was then further diluted with the mixture of $500 \mathrm{~mL}$ distilled water and $20 \mathrm{~mL}$ of hydrogen peroxide (5\%). The mixture was then left overnight which was followed by washing with $\mathrm{HCl}$ several times and then centrifuged at $4000 \mathrm{rpm}$ to remove the upper solution. This was then followed by washing with the distilled water several times to obtain the graphene oxide solution.

\section{Colloidal Hydroxyapatite preparation}

Colloidal Hydroxyapatite (HA) was prepared by the following method. Briefly, $14 \mathrm{~g}$ ammonium dihydrogen phosphate and $24 \mathrm{~g}$ Calcium nitrate was first dissolved in $50 \mathrm{~mL}$ ethanol, respectively to make the ammonium dihydrogen phosphate stock and Calcium nitrate stock. Then Calcium nitrate stock was transferred to the $250 \mathrm{~mL}$ round bottom flask with the magnetic stirring. Ammonium dihydrogen phosphate 

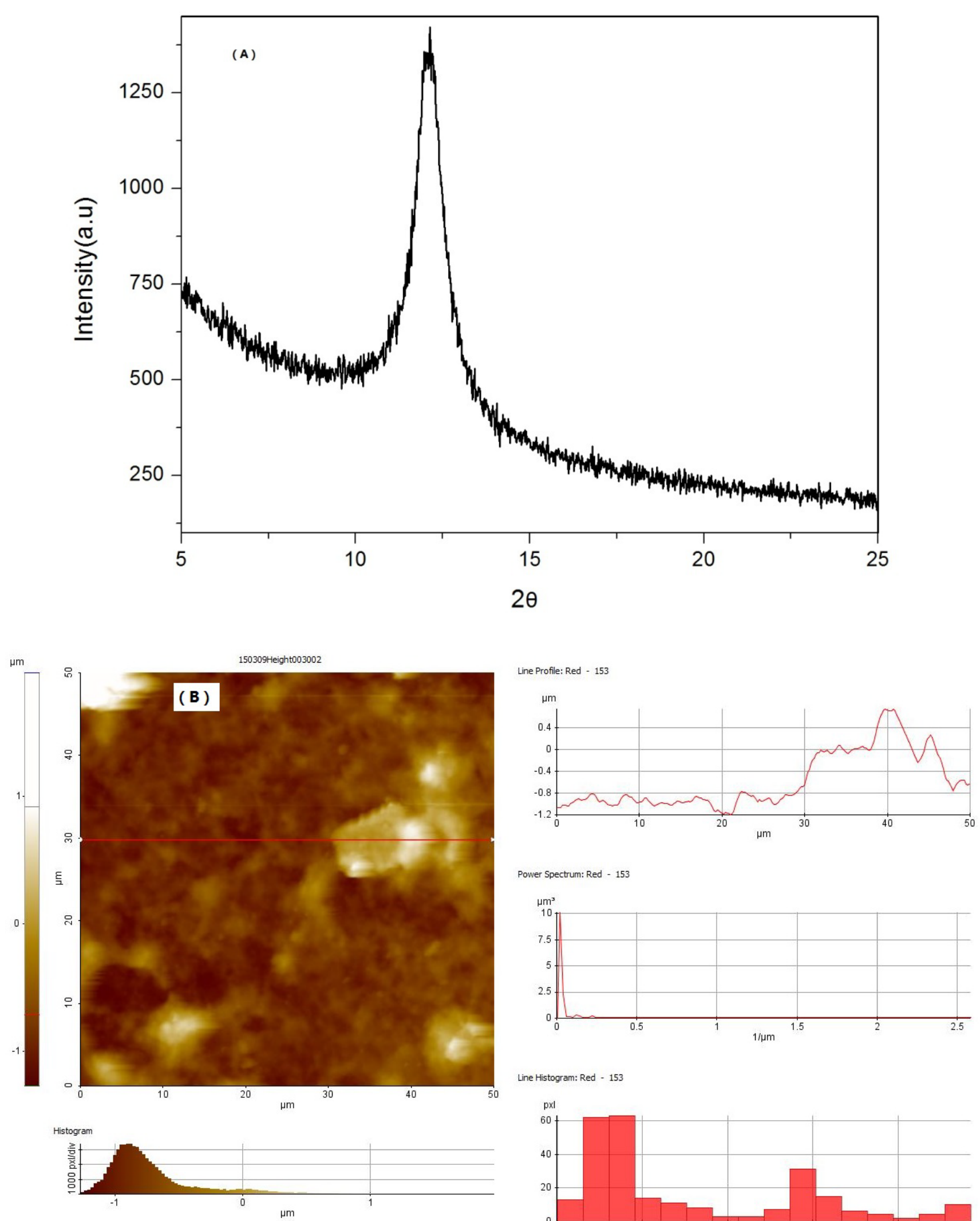

Line Histogram: Red - 153

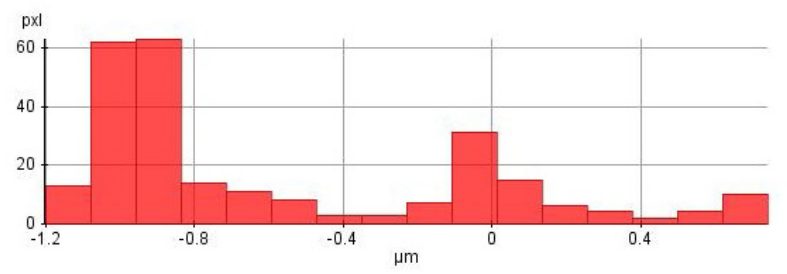

Figure 1. XRD spectra of GO (A) and AFM observations of GO (B). 


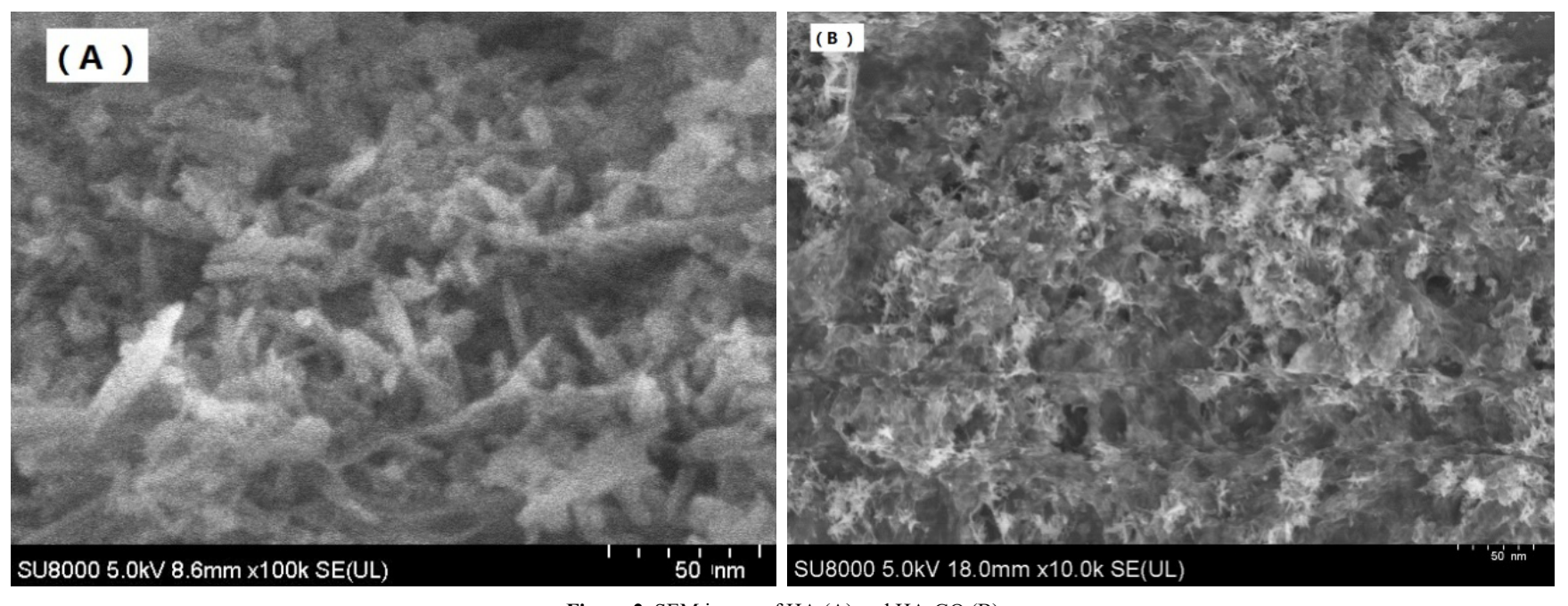

Figure 2. SEM image of HA (A) and HA-GO (B).

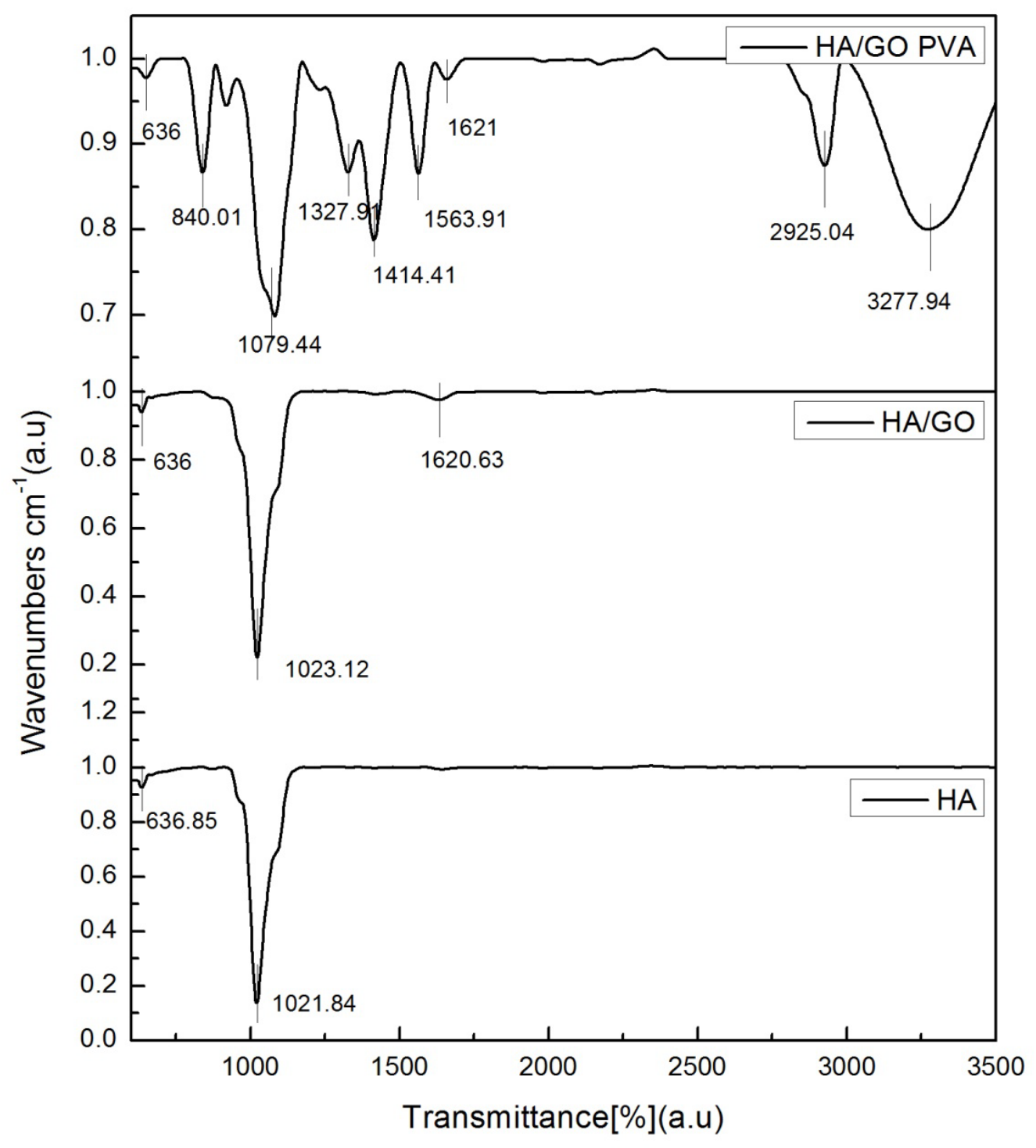

Figure 3. FT-IR spectra of HA and HA-GO 

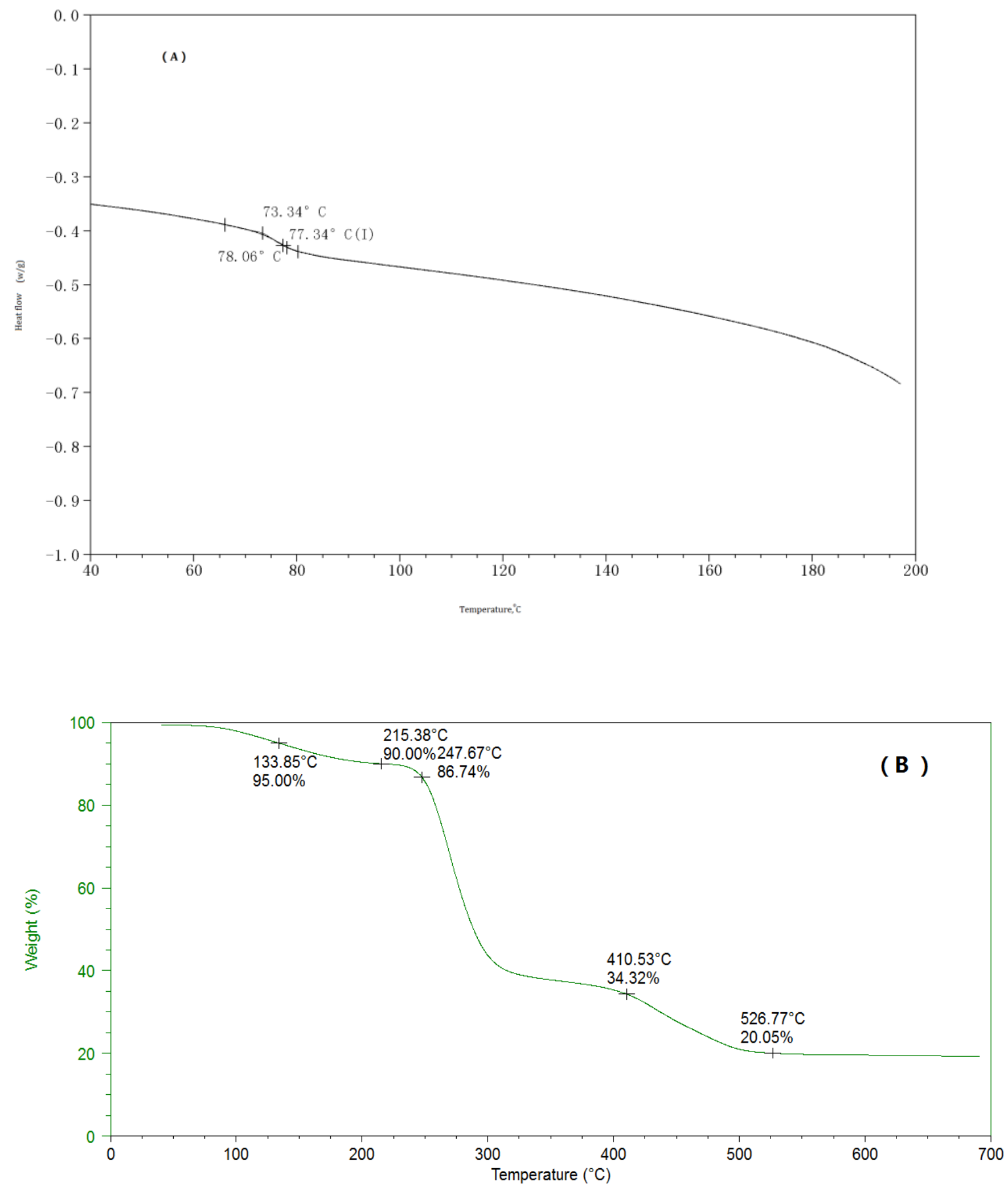

Figure 4. DSC (A) and TGA (B) of the HA-GO-PVA nanocomposite. 


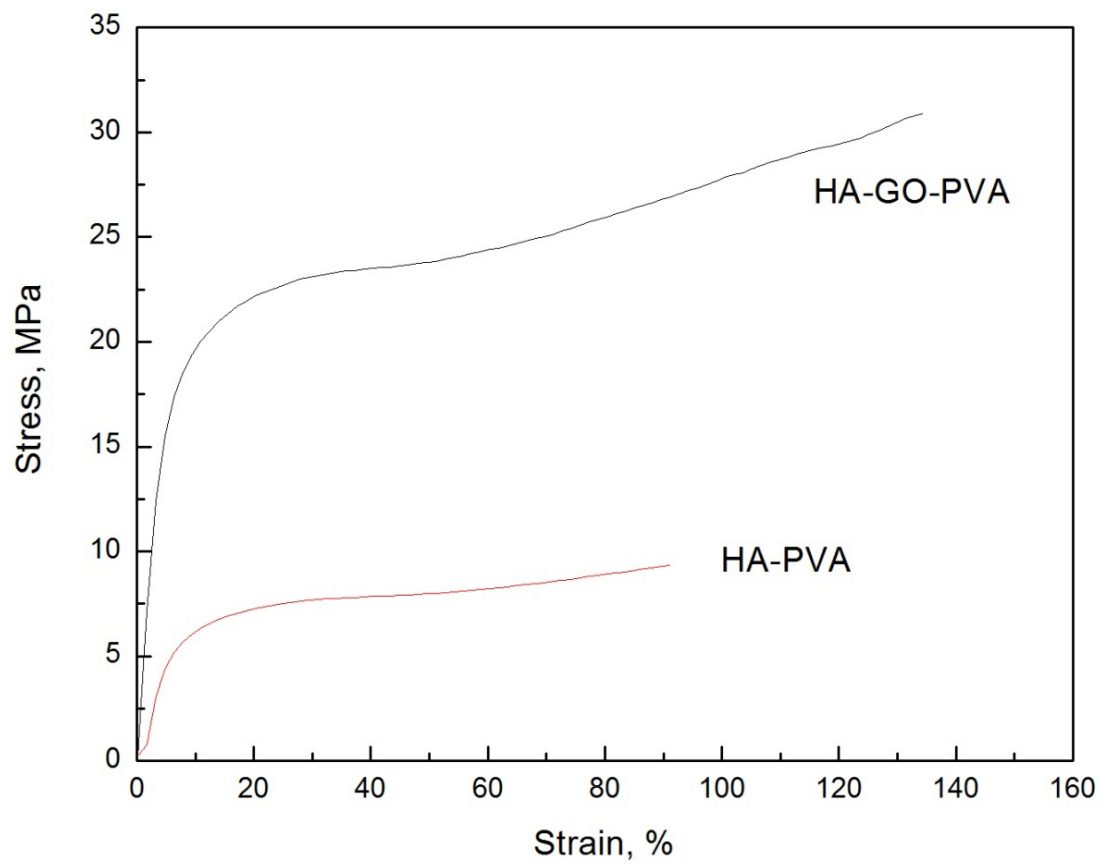

Figure 5. Stress-strain curve of the HA-PVA and HA-GO-PVA nanocomposite.
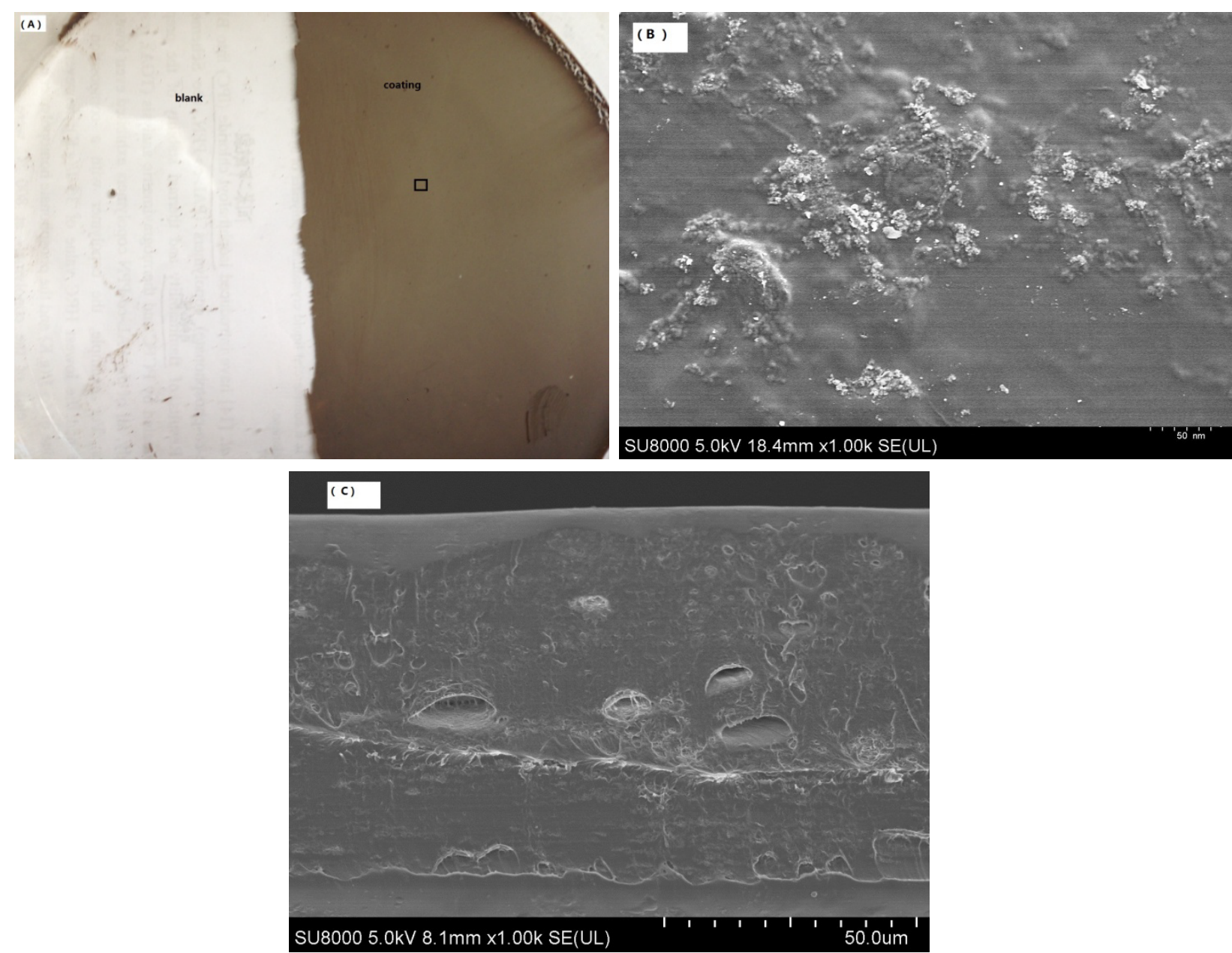

Figure 6. HA-GO-PVA nanocomposite coating (A) and SEM image of THE surface (B) and cross section (C). 
stock was added into the Calcium nitrate stock in the flask dropwisely at $60{ }^{\circ} \mathrm{C}$ with a condenser. Then the mixture was stirring for 24 hours before increasing the temperature to $90^{\circ} \mathrm{C}$ for another 4 hours. The final so-gel like solution was centrifuged and dispersed in the $\mathrm{deH}_{2} \mathrm{O}$ with ultrasonic disperse to get the HA colloidal solution.

\section{HA-GO-PVA nanocomposites}

$20 \mathrm{~mL} \mathrm{HA}$ colloidal solution and $20 \mathrm{~mL}$ graphene oxide solution was mixed in the round bottom flask under magnetic stirring at room temperature for 24 hours. And then 10g PVA was dissolved in 30 $\mathrm{mL} \mathrm{deH}_{2} \mathrm{O}$ at $60^{\circ} \mathrm{C}$ and then this PVA solution was dropped into the flask to mix with the HA and GO solution for another 4 hours. The resultant mixture was then shaped for the mechanical tests or used for the coating on the glass plates.

\section{Characteristic}

The GO was tested by FR-IR with PerkinElmer Universal STR Sampling PerkinElmer Spectrum 100 Series and the samples were directly loaded onto the plate. All the samples for XRD were determined by powder X-ray diffraction (XRD) analyses with Hilton brooks modified Philips X-ray diffractometer $(\mathrm{Cu} \mathrm{Ka}, \lambda=1.5418 \AA)$ and generated at $30 \mathrm{kV}, 30 \mathrm{~mA}$. Scattering angles were ranged from $1^{\circ}$ to $60^{\circ}$.

\section{Conclusions}

The results in this research showed that the GO and HA can be well mixed and the HA nanoparticles can be well dispersed in the GO. Due to the $-\mathrm{OH}$ hydrophilic surfaces of the $\mathrm{HA}$ and $\mathrm{GO}$, polymer matrix PVA is compatible with HA and GO to form composite materials. The addition of the HA and GO showed good compatibility in the mechanical strength and the GO can improve the mechanical strength. This material is a promising biomedical coating for the bone implant other surgery applications.

\section{Acknowledgement}

This project was supported by the Scientific Research Initial funding for the advanced talent of Janghan University (08010001, 06750001), Basic Research Project of Wuhan City (2015011701011593), 4th Yellow Crane Talent Programme of Wuhan City (08010004), Hubei Province Innovative Young Research Team in Universities (T201318), The Key Project of Natural Science Foundation of Hubei Province (2014CFA098) and the open fund the Key lab (JDGD201613).

\section{Conflicts of interest}

The authors declare no conflict of interest.

\section{References}

1. Klimavicius V, Kareiva A, Balevicius V (2014) Solid-state NMR study of hydroxyapatite containing smorphous phosphate phase and nanostructured hydroxyapatite: cut-off averaging of CP-MAS kinetics and size profiles of spin clusters. J Phys Chem C 118: 28914-28921.

2. Sun JP, Dai JH, Song Y, Wang Y, Yang, R (2014) Affinity of the interface between hydroxyapatite (0001) and titanium (0001) surfaces: a first-principles investigation. ACS Appl Mater Interfaces 6: 20738-20751. [Crossref]

3. Zhang QM, Serpe MJ (2014) Synthesis, characterization, and antibacterial properties of a hydroxyapatite adhesive block copolymer. Macromolecules 47: 8018-8025.

4. Jensen ACS, Ibsen CJS, Sutherland D, Birkedal H (2014) Transparent aggregates of nanocrystalline hydroxyapatite. Crystal Growth \& Design 14: 6343-6352.

5. Wang M, Gao JY, Shi C, Zhu YC, Zeng, Y, et al. (2014) Facile one-pot synthesis of oriented pure hydroxyapatite with hierarchical architecture by topotactic conversion. Crystal Growth \& Design 14: 6459-6466.

6. Zhao WL, Xu ZJ, Yang Y, Sahai N (2014) Surface energetics of the hydroxyapatite nanocrystal-water interface: a molecular dynamics study. Langmuir 30: 13283-13292.

7. Wang YW, Christenson HK, Meldrum FC (2014) Confinement increases the lifetimes of hydroxyapatite precursors. Chem Mater 26: 5830-5838.

8. Hu Y, Gu XY, Yang Y, Huang JA, Hu M, et al. (2014) Facile fabrication of poly (1-lactic acid)-grafted hydroxyapatite/poly (lactic-co-glycolic acid) scaffolds by pickering high internal phase emulsion templates. ACS Appl Mater Interfaces 6:17166-17175.

9. Salarian M, Xu WZ, Wang ZQ, Sham TK, Charpentier PA(2014) Hydroxyapatite-TiO2based nanocomposites synthesized in supercritical $\mathrm{CO} 2$ for bone tissue engineering: physical and mechanical properties. ACS Appl Mater Interfaces 6: 16918-16931.

10. Tampieri A, Iafisco M, Sandri M, Panseri S, Cunha C, et al. (2014) Magnetic bioinspired hybrid nanostructured collagen-hydroxyapatite scaffolds supporting cell proliferation and tuning regenerative process. ACS Appl Mater Interfaces 6: 15697-15707.

11. McManamon C, Silva JP, Power J, Garcia SR, Morris MA, et al. (2014) Interfacial characteristics and determination of cohesive and adhesive strength of plasma-coated hydroxyapatite via nanoindentation and microscratch techniques. Langmuir 30: 1141221140. [Crossref]

12. Yang YS, Wu QZ, Wang M, Long J, Mao Z, et al. (2014) Hydrothermal synthesis of hydroxyapatite with different morphologies: influence of supersaturation of the reaction system. Crystal Growth \& Design 14: 4864-4871.

13. Klibanski SB, Yalin D, Shenker M (2014) Comment on "Formations of hydroxyapatite and inositol hexakisphosphate in poultry litter during the composting period: sequential fractionation, P K-edge XANES and solution 31P NMR investigations". Environ Sci Technol 48: 9955-9956.

14. Barros AA, Aroso IM, Silva TH, Mano JF, Duarte ARC, et al. (2014) Surface modification of silica-based marine sponge bioceramics induce hydroxyapatite formation. Crystal Growth \& Design 14: 4545-4552.

15. Uskokovifi V, Desai TA (2014) Does translational symmetry matter on the micro scale? Fibroblastic and osteoblastic interactions with the topographically distinct poly (e-caprolactone)/ hydroxyapatite thin films. ACS Appl Mater Interfaces 6: 13209-13220.

16. Liu M, Liu H, Sun SF, Li XJ, Zhou YM, et al. (2014) Correction to multifunctional hydroxyapatite/Na (Y/Gd) F4:Yb3+, Er3 + composite fibers for drug delivery and dual modal imaging. Langmuir 30: 7621.

17. Nathanael AJ, Yuvakkumar R, Hong SI, Oh TH (2014) Novel zirconium nitride and hydroxyapatite nanocomposite coating: detailed analysis and functional properties. ACS Appl Mater Interfaces 6: 9850-9857.

18. Li DW, Sun HZ, Jiang LM, Zhang K, Liu WD, et al. (2014) Enhanced biocompatibility of PLGA nanofibers with gelatin/nano-hydroxyapatite bone biomimetics incorporation. ACS Appl Mater Interfaces 6: 9402-9410. [Crossref]

19. Garcia SD, Osman MB, Krafft JM, Casale S, Thomas C, et al. (2014) Identification of surface basic sites and acid-base pairs of hydroxyapatite. JPhys Chem C 118: 12744-12757.

20. Liao CY, Zhou J (2014) Replica-exchange molecular dynamics simulation of basic fibroblast growth factor adsorption on hydroxyapatite. J Phys Chem B 118: 5843-5852.

21. Bhat SS, Waghmare UV, Ramamurty U (2014) First-principles study of structure, vibrational, and elastic properties of stoichiometric and calcium-deficient hydroxyapatite. Crystal Growth \& Design 14: 3131-3141.

22. Kanno CM, Sanders RL, Flynn SM, Lessard G, Myneni SCB (2014) Novel apatitebased sorbent for defluoridation: synthesis and sorption characteristics of nano-microcrystalline hydroxyapatite-coated-limestone. Environ Sci Technol 48: 5798-5807.

23. Hashimoto Y, Takamoto A, Kikkawa R, Murakami K, Yamaguchi N (2014) Formations of hydroxyapatite and inositol hexakisphosphate in poultry litter during the composting period: sequential fractionation, PK-edge XANES and solution 31P NMR investigations. Environ Sci Technol 48: 5486-5492.

24. Tay CY, Fang W, Setyawati MI, Chia SL, Tan KS, et al. (2014) Nano-hydroxyapatite and nano-titanium dioxide exhibit different subcellular distribution and apoptotic profile in human oral epithelium. ACS Appl Mater Interfaces 6: 6248-6256.

Copyright: (C2017 Cao YC. This is an open-access article distributed under the terms of the Creative Commons Attribution License, which permits unrestricted use, distribution, and reproduction in any medium, provided the original author and source are credited. 